\begin{tabular}{|c|l|}
\hline Title & Photo-A ctivatable A kt Probe: A New Tool to Study the A kt-Dependent Phy siopathology of Cancer Cells \\
\hline Author(s) & Haga, Sanae; Ozawa, Takeaki; Morita, Naoki; A sano, Mami; Jin, Shigeki; Y imin; Ozaki, Michitaka \\
\hline Citation & $\begin{array}{l}\text { Oncology research, 26(3), 467-472 } \\
\text { https://doi.org/L0.3727/096504017X 15040166233313 }\end{array}$ \\
\hline Issue Date & 2018.0410 \\
\hline Doc URL & http://hdl.handle.net/2115/70690 \\
\hline Rights(URL) & http://creativecommons.org/icenses/by-nc/3.0/ \\
\hline Type & article \\
\hline File Information & s13.pdf \\
\hline
\end{tabular}

Instructions for use 


\title{
Photo-Activatable Akt Probe: A New Tool to Study the Akt-Dependent Physiopathology of Cancer Cells
}

\author{
Sanae Haga, ${ }^{*}$ Takeaki Ozawa, $\dagger$ Naoki Morita, $\ddagger$ Mami Asano, $§$ Shigeki Jin,I \\ Yimin,\# and Michitaka Ozaki*§凹
}

\begin{abstract}
*Department of Biological Response and Regulation, Faculty of Health Sciences, Hokkaido University, Sapporo, Japan $\dagger$ Department of Chemistry, School of Science, The University of Tokyo, Tokyo, Japan $\$$ Bioproduction Research Institute, National Institute of Advanced Industrial Science and Technology (AIST), Sapporo, Hokkaido, Japan

§Laboratory of Molecular and Functional Bioimaging, Faculty of Health Sciences, Hokkaido University, Sapporo, Japan I[Core Research Laboratory, Faculty of Health Sciences, Hokkaido University, Sapporo, Japan \#Department of Advanced Medicine, Graduate School of Medicine, Hokkaido University, Sapporo, Japan
\end{abstract}

\begin{abstract}
Akt is commonly overexpressed and activated in cancer cells and plays a pivotal role in cell survival, protection, and chemoresistance. Therefore, Akt is one of the target molecules in understanding characters of cancer cells and developing anticancer drugs. Here we examined whether a newly developed photo-activatable Akt (PA-Akt) probe, based on a light-inducible protein interaction module of plant cryptochrome2 (CRY2) and cryptochrome-interacting basic helix-loop-helix (CIB1), can regulate Akt-associated cell functions. By illuminating blue light to the cells stably transfected with PA-Akt probe, CRY2-Akt (a fusion protein of CRY2 and Akt) underwent a structural change and interacted with Myr-CIBN (myristoylated N-terminal portion of CIB1), anchoring it at the cell membrane. Western blot analysis revealed that S473 and T308 of the Akt of probeAkt were sequentially phosphorylated by intermittent and continuous light illumination. Endogenous Akt and GSK-3 $\beta$, one of the main downstream signals of Akt, were also phosphorylated, depending on light intensity. These facts indicate that photo-activation of probe-Akt can activate endogenous Akt and its downstream signals. The photo-activated Akt conferred protection against nutritional deprivation and $\mathrm{H}_{2} \mathrm{O}_{2}$ stresses to the cells significantly. Using the newly developed PA-Akt probe, endogenous Akt was activated easily, transiently, and repeatedly. This probe will be a unique tool in studying Akt-associated specific cellular functions in cancer cells and developing anticancer drugs.
\end{abstract}

Key words: Photo-activatable probe; Akt; Nutritional deprivation; Oxidative stress

\section{INTRODUCTION}

Akt is expressed ubiquitously and constitutively in various types of cells and organs and is known to be a key molecule in cell survival/protection in noncancerous as well as cancerous cells ${ }^{1-10}$. Akt sends various kinds of signals which are important for antioxidation ${ }^{7,8}$, antiapoptosis $^{8,9}$, cell proliferation/growth ${ }^{6}$, and sugar/lipid metabolism $^{5}$ in cells. In cancer cells, Akt is often upregulated at the level of both gene and protein expression and contributes to cell survival by conferring resistance against various physical and biological stresses ${ }^{6-10}$. Therefore, Akt has been a good target for the development of anticancer drugs $^{11,12}$.

Recently, a unique optic probe to activate Akt has been developed, potentially activating endogenous Akt in various cells ${ }^{13}$. The photo-activatable Akt (PA-Akt) probe is based on a light-inducible protein interaction module of Arabidopsis thaliana cryptochrome2 (CRY2) and cryptochrome-interacting basic helix-loop-helix (CIB1). In the cells, CRY2 is expressed as a fusion protein with Akt (CRY2-Akt), and N-terminal portion of CIB1 (CIBN) is myristoylated (Myr-CIBN), enabling CIBN to anchor at the cell membrane. By illuminating light to the cells, CRY2 of CRY2-Akt, interacting with CIBN of Myr-CIBN, anchors Akt of CRY2-Akt at the cell membrane. Akt can then be activated by kinases located at the cell membrane.

In the present study, we proved that the PA-Akt probe can activate the endogenous Akt of cells and protect cells against biological stresses. Light illumination induced 
Akt phosphorylation of the PA-Akt probe, which further phosphorylated endogenous Akt and its downstream signals, conferring resistance against malnutrition and oxidative stresses. This will provide a unique tool to analyze Akt-specific cellular effects and evaluate various drugs targeting Akt.

\section{MATERIALS AND METHODS}

\section{Cell Culture and Reagents}

The $\alpha$ mouse liver 12 (AML12) cells, established from hepatocytes from a mouse transgenic for human transforming growth factor- $\alpha$ (TGF- $\alpha$ ), express high levels of human TGF- $\alpha$ and lower levels of mouse TGF- $\alpha$ (ATCC, Manassas, VA, USA). AML12 cells were maintained at $37^{\circ} \mathrm{C}$ in $5.0 \% \mathrm{CO}_{2}$ in Dulbecco's modified Eagle's medium (high-glucose DMEM; 450 mg/dl glucose; Sigma-Aldrich, St. Louis, MO, USA) supplemented with $10 \%$ fetal bovine serum (FBS). For nutritional deprivation, AML12 cells were cultured in low-glucose DMEM (150 mg/dl glucose) without FBS.

\section{Generation of AML12 Cells Stably Expressing the Photo-Activatable Probe for Akt Activation}

The expression vectors encoding Myr-Venus-CIBNmyc and CRY2-mCherry-Akt-V5 (Fig. 1A) ${ }^{13}$ were separately introduced into AML12 cells using Lipofectamine 2000 reagent (Thermo Fisher Scientific, Waltham, MA, USA), in accordance with the manufacturer's instruction. Cells stably expressing these two proteins were obtained after selection with G418 and hygromycin B (Nacalai Tesque Inc., Kyoto, Japan) for 2 weeks.

\section{Light Illumination to Cells}

LED pulse irradiation device (model No. IL-03; iTest Co., Ltd., Osaka, Japan) was used to illuminate cells with blue light (wavelength: $450 \mathrm{~nm}$, pulse drive frequency: $800 \mathrm{~Hz}$ ). This generates various light intensities of 0.7 to $7.7 \mathrm{~mW} / \mathrm{cm}^{2}$ at the sample points.

\section{Immunoprecipitation and Western Blot Analysis}

The detailed procedure of immunoprecipitation and Western blot to detect endogenous Akt and CRY2-Akt (Probe-Akt) is provided in Supplementary Fig. 1 (available at http://prometheus-lsi.com/wp-content/uploads/ 2017/08/Suppl-Fig-1-ORM-A-1577-Haga-S-et-al.jpg). The following were used as primary antibodies: Akt/ phospho-Akt and glycogen synthase kinase-3 (GSK-3)/ phospho-GSK-3 (Cell Signaling Technology, Boston, MA, USA). Endogenous Akt and CRY2-Akt (Probe-Akt) were detected at 60 and $130 \mathrm{kDa}$, respectively (Supplementary Fig. 2, available at http://prometheus-lsi.com/wp-content/ uploads/2017/08/Suppl-Fig-2-ORM-A-1577-Haga-Set-al.jpg), because CRY2-Akt is much heavier than endogenous $\mathrm{Akt}^{13}$.

\section{Evaluation of Hepatocyte Cell Death}

Hepatocyte cell death was evaluated by measuring lactate dehydrogenase (LDH) release in culture media by LDH Cytotoxicity Detection Kit (Takara Bio Inc., Shiga, Japan) and observing attached cells by light microscopy. LDH detection was performed according to the manufacturer's instructions. Briefly, the cell-free culture medium (cells removed by centrifugation) was added to an equal volume of reaction mixture (catalyst and dye solution) and incubated at room temperature for $30 \mathrm{~min}$. Then the samples were measured by absorbance at $490 \mathrm{~nm}$.

\section{Statistical Analysis}

All results were expressed as means \pm standard error of the mean (SEM). Statistical analyses were performed with Fisher's test, and values of $p<0.05$ were considered significant.

\section{RESULTS AND DISCUSSION}

The Activation Mechanism of PA-Akt Probe by Blue Light in Cells

Figure 1A shows the constructs of two components of the PA-Akt probe. The PA-Akt probe consists of CRY2Akt (CRY2-mCherry-Akt tagged with V5) and MyrCIBN (Myr-Venus-CIBN tagged with myc), which were stably transfected to AML12 cells. mCherry and Venus tagged to the PA-Akt probe are fluorophores that provide red and green colors, respectively. Therefore, they can be used as the tracers of these two molecules of the PA-Akt probe. By fluorescent microscopic observation, CRY2mCherry-Akt-V5 was expressed in the cytosol (red). MyrVenus-CIBN-myc was expressed/localized at the cell membrane because it is myristoylated (green) (Fig. 1B). Illumination to the cells with blue light (450-nm wavelength) induced interaction of CRY2 with CIBN, generating a "yellow" signal [mixed red (CRY2) and green $(\mathrm{CIBN})]$. This indicates that the Akt of CRY2-mCherryAkt-V5 was anchored near the cell membrane. These data indicate that the Akt of the probe can be moved to the cell membrane by illuminating with blue light, and Akt is then expected to be phosphorylated/activated by the kinases located at the cell membrane.

\section{Phosphorylation of the Probe-Akt and Endogenous Akt/GSK-3 of AML12 Cells by Blue Light Illumination}

Next, we studied whether Akt of the PA-Akt probe (probe-Akt) and endogenous Akt are phosphorylated/activated in AML12 cells stably transfected with the PA-Akt probe (AML12/PA-Akt cells). AML12/PA-Akt cells were stimulated intermittently and continuously illuminated by blue light $(5 \mathrm{~s} / \mathrm{min} / \mathrm{set})$. The illumination of blue light promptly induced phosphorylation of probe-Akt at S473 and T308 sequentially by light illumination (Fig. 2A). 
A Myr-CIBN

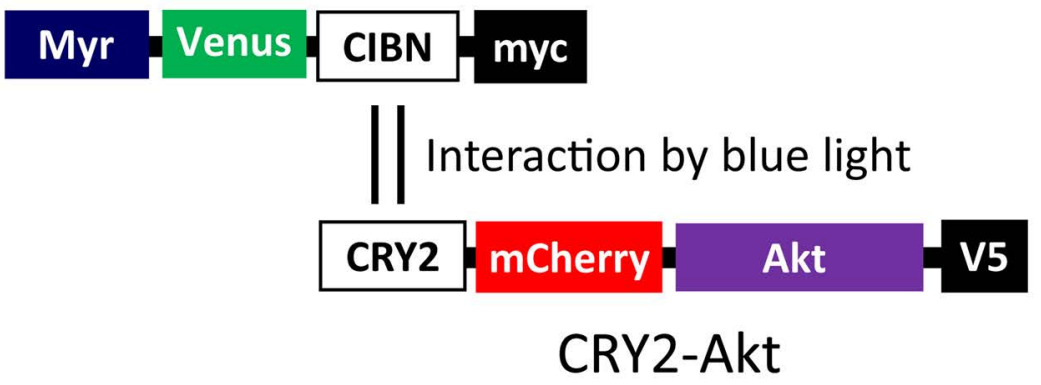

B
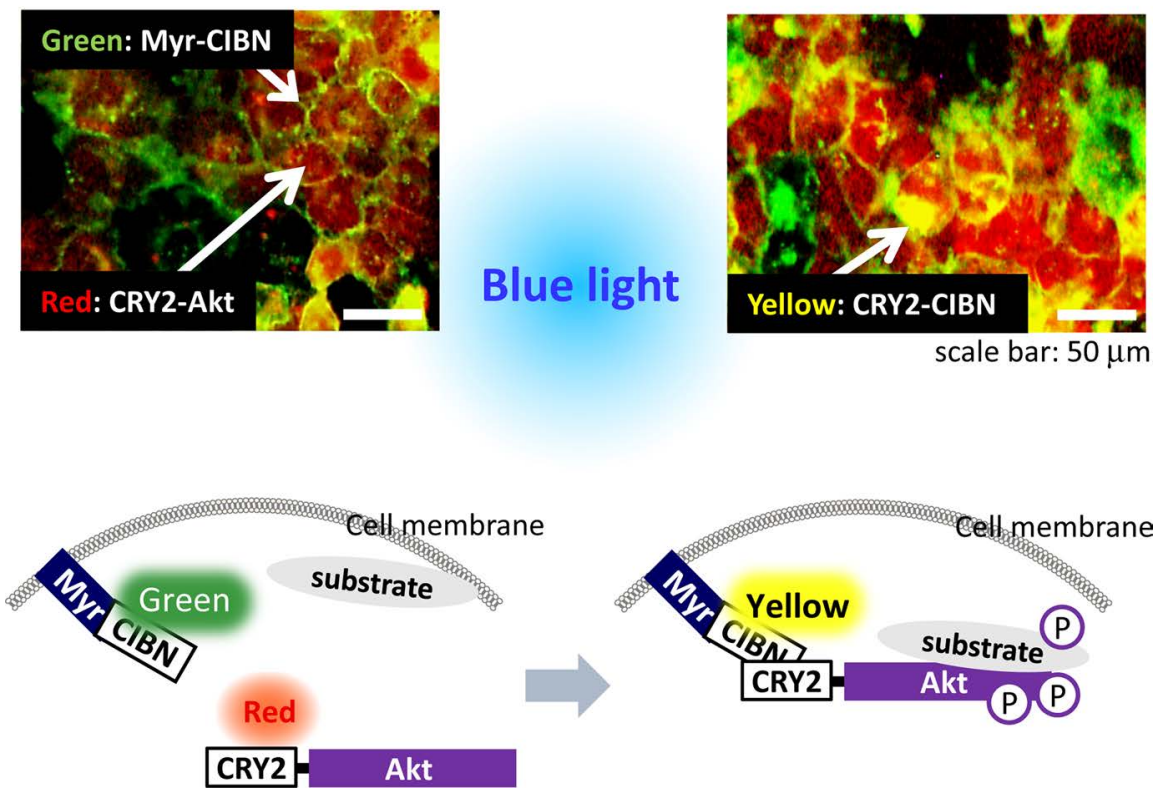

Figure 1. Generation of cells stably transfected with photo-activatable Akt (PA-Akt) probe. (A) CRY2-mCherry-Akt tagged with V5 and Myr-Venus-CIBN tagged with myc were stably transfected into AML12 cells (AML12/PA-Akt cells). mCherry and Venus are fluorophores and provide red and green colors, respectively. Myr stands for myristoylation signal. Plant cryptochrome 2 (CRY2) and the $\mathrm{N}$-terminal of cryptochrome-interacting basic helix-loop-helix (CIBN) of a light-inducible protein interaction module work together under blue light illumination. (B) The photomicrographs and schematic illustration demonstrate the mechanism as to how the light affects the two molecules and activates Akt in cells. CRY2-mCherry-Akt-V5 and Myr-Venus-CIBN-myc were expressed/localized in the cytosol (red) and cell membrane (green), respectively. By illuminating the cells with blue light (450-nm wavelength, $3.4 \mathrm{~mW} / \mathrm{cm}^{2}$ ), cytosolic CRY2-mCherry-Akt-V5 moved to the membrane and interacted with Myr-Venus-CIBN-myc, thereby providing a "yellow" signal mixed with red and green colors.

Phosphorylation of the probe-Akt at T308 occurred later than at S473. Phosphorylation of T308 is considered to be more important for Akt activation ${ }^{4}$, so 10 sets of light illumination may be required for sufficient Akt activity.

Therefore, we performed the following experiments at 10 sets of light illumination with various light intensities. The light illumination induced phosphorylation of endogenous Akt, depending on the light intensity (Fig. 2B). This also phosphorylated GSK-3 $\beta$, one of the main downstream signals of Akt. These data indicate that the activation of the PA-Akt probe by light illumination activates endogenous Akt and its downstream signals in cells and is therefore expected to regulate Akt-associated cellular functions.

\section{Photo-Activated Akt and Cellular Resistance Against Stresses}

We next challenged the AML12/PA-Akt cells with various stresses, such as nutritional deprivation and oxidative stress. Nutrition was deprived by the replacement 
A

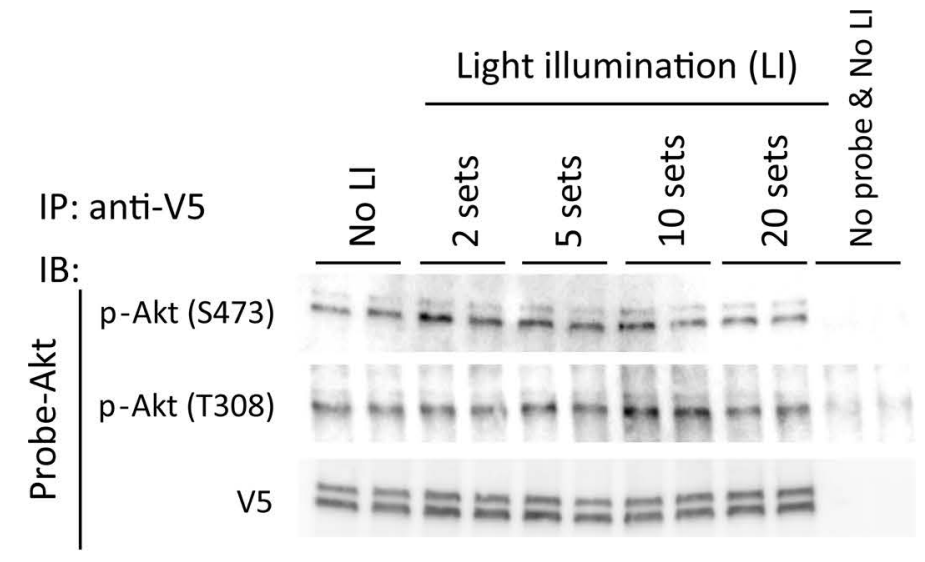

Light illumination : $5 \mathrm{sec} / \mathrm{min} / \mathrm{set}$
p-Akt(S)/V5

p-Akt(T)/V5

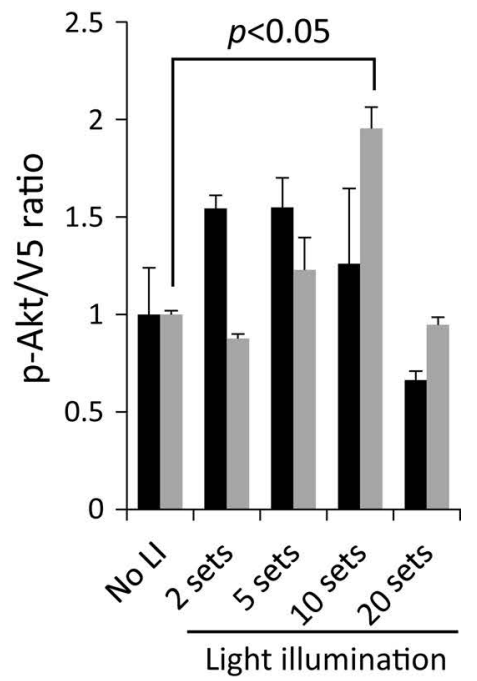

B

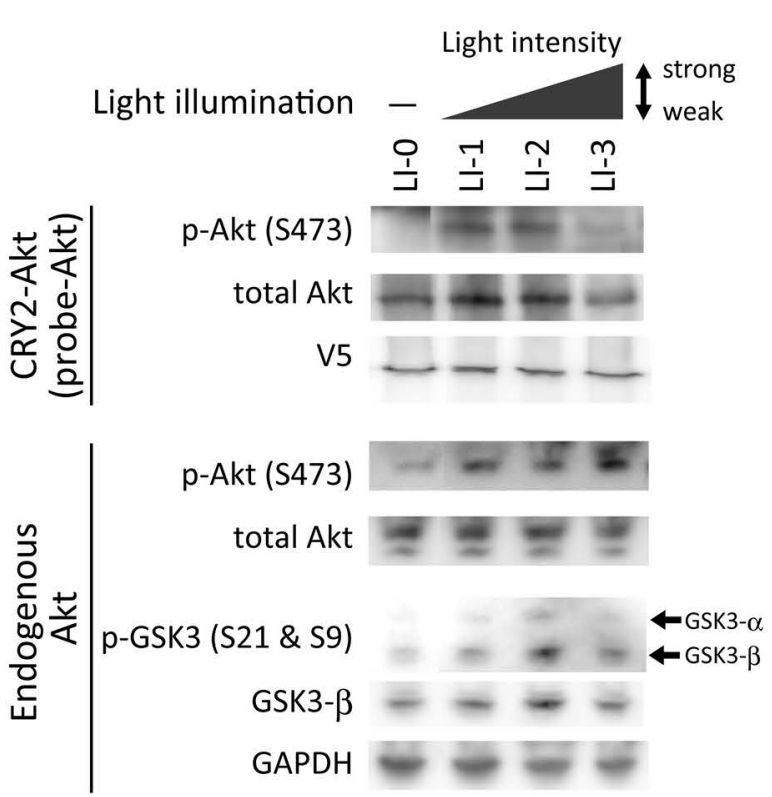

Light illumination : $5 \mathrm{sec} / \mathrm{min} \times 10 \mathrm{set}(10 \mathrm{~min})$
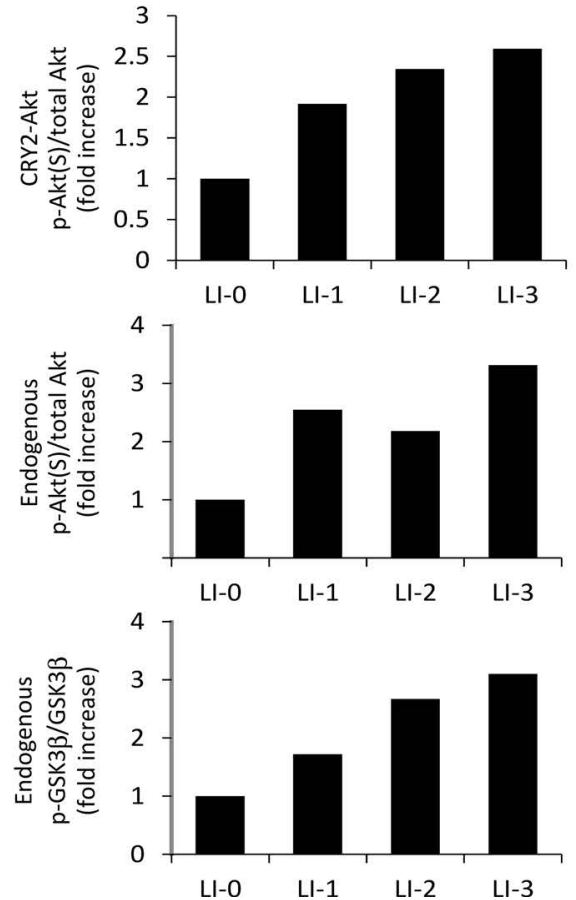

Figure 2. Phosphorylation/activation of Akt of the PA-Akt (probe-Akt) and endogenous Akt/GSK-3 by light illumination. (A) Western blot analysis showed the expression of probe-Akt at $130 \mathrm{kDa}$ (see Supplementary Fig. 2, available at http://prometheus-lsi.com/wpcontent/uploads/2017/08/Suppl-Fig-2-ORM-A-1577-Haga-S-et-al.jpg). Probe-Akt was sequentially phosphorylated at S473 and T308 in AML12/PA-Akt cells by light illumination ( $1 \mathrm{set} ; 5 \mathrm{~s} / \mathrm{min}, 3.4 \mathrm{~mW} / \mathrm{cm}^{2}$ ). Light was not illuminated in "No LI." IP and IB stand for immunoprecipitation and immunoblot, respectively. (B) Endogenous Akt was detected at $60 \mathrm{kDa}$. Light illumination induced phosphorylation of endogenous Akt and glycogen synthase kinase $3 \beta$ (GSK-3 $\beta$ ), depending on the light intensity. The conditions of light illumination were $5 \mathrm{~s} / \mathrm{min} \times 10$ sets at the light intensities of $2.3,4.3$, and $7.0 \mathrm{~mW} / \mathrm{cm}^{2}$ for LI-1, LI-2, and LI-3, respectively. Light was not illuminated to "LI-0." GAPDH, glyceraldehyde 3-phosphate dehydrogenase. 
A

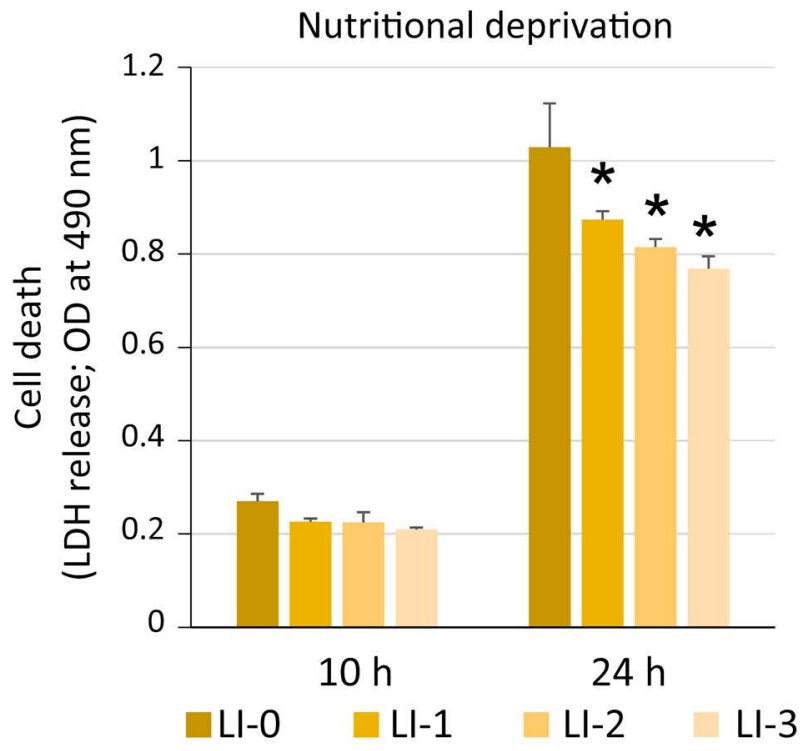

B

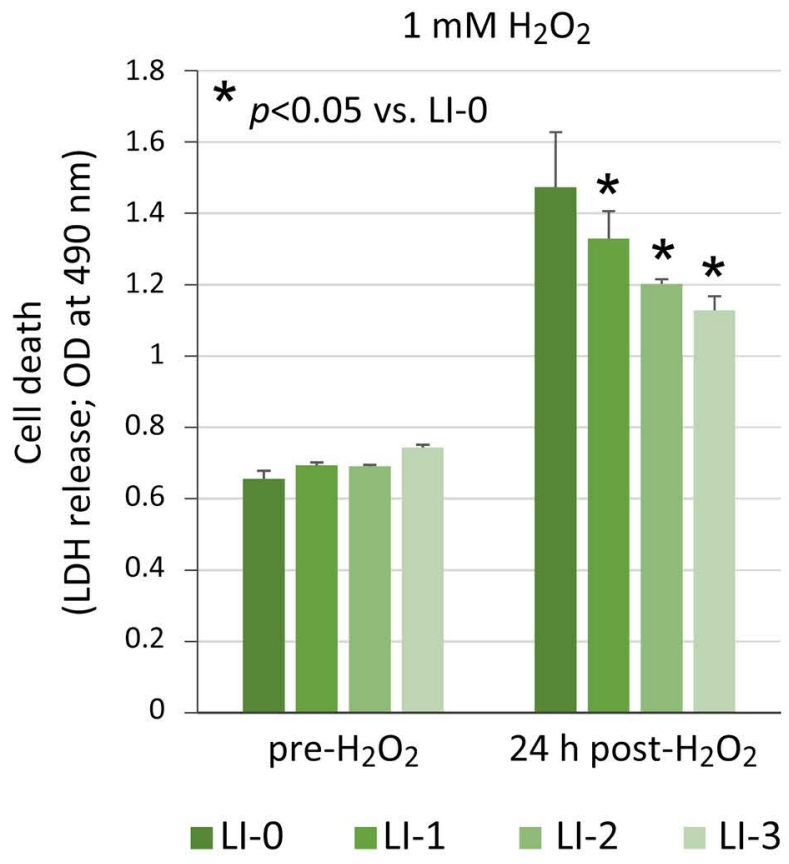

* $p<0.05$ vs. LI-0
Light illumination

\begin{tabular}{|l|l|}
\hline LI-0 & None \\
\hline LI-1 & $1 \mathrm{sec} / \mathrm{min} \times 10$ \\
\hline LI-2 & $2 \mathrm{sec} / \mathrm{min} \times 10$ \\
\hline LI-3 & $5 \mathrm{sec} / \mathrm{min} \times 10$ \\
\hline
\end{tabular}

Photomicrograph (24 h post $-\mathrm{H}_{2} \mathrm{O}_{2}$ insult)
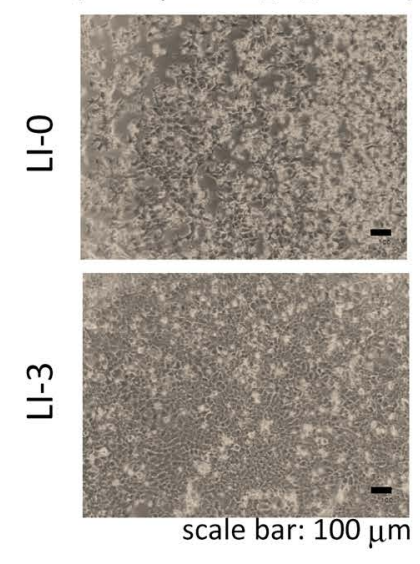

Light illumination

\begin{tabular}{|l|l|}
\hline LI-0 & None \\
\hline LI-1 & $12 \mathrm{~h}$ prior to $\mathrm{H}_{2} \mathrm{O}_{2}$ \\
\hline $\mathrm{LI}-2$ & $6 \mathrm{~h}$ prior to $\mathrm{H}_{2} \mathrm{O}_{2}$ \\
\hline $\mathrm{LI}-3$ & $0 \mathrm{~h}$ prior to $\mathrm{H}_{2} \mathrm{O}_{2}$ \\
\hline
\end{tabular}

Figure 3. Prosurvival effects of photo-activated Akt in AML12/PA-Akt cells. (A) AML12/PA-Akt cells were protected against 24-h nutritional deprivation [lactate dehydrogenase (LDH) release and cell detachment]. For nutritional deprivation, Dulbecco's modified Eagle's medium (DMEM; $450 \mathrm{mg} / \mathrm{dl}$ glucose) with $10 \%$ fetal bovine serum (FBS) was replaced to DMEM (150 mg/dl glucose) without FBS. Conditions of light illumination: 1 (LI-1), 2 (LI-2), and 5 (LI-3) s/min $\times 10$ times with the light intensity of $3.4 \mathrm{~mW} / \mathrm{cm}^{2}$. Light was not illuminated in "LI-0." (B) AML12/PA-Akt cells were protected against hydrogen peroxide $\left(\mathrm{H}_{2} \mathrm{O}_{2}\right)(\mathrm{LDH}$ release and cell detachment). Light was not illuminated to "LI-0." Light was illuminated $12 \mathrm{~h}$ (LI-1), $6 \mathrm{~h}$ (LI-2), or $0 \mathrm{~h}$ (LI-3) prior to $\mathrm{H}_{2} \mathrm{O}_{2}$ treatment $(1 \mathrm{mM})$. Light intensity: $3.4 \mathrm{~mW} / \mathrm{cm}^{2}$. Results are expressed as mean \pm standard error of the mean (SEM) of three independent experiments, with $p<0.05$ considered significant. 
of DMEM (450 mg/dl glucose) with $10 \%$ FBS to DMEM (150 mg/dl glucose) without FBS. Twenty-four hours of nutritional deprivation induced marked cell death of AML12/PA-Akt cells (Fig. 3A). By illuminating light prior to nutritional deprivation, cell death was significantly suppressed depending on the time of light illumination. As an oxidative stress to cells, hydrogen peroxide $\left(\mathrm{H}_{2} \mathrm{O}_{2}\right)$ was administered to AML12/PA-Akt cells. $\mathrm{H}_{2} \mathrm{O}_{2}$ (1 mM) was enough to induce significant cell death $24 \mathrm{~h}$ after $\mathrm{H}_{2} \mathrm{O}_{2}$ treatment. AML12/PA-Akt cells were significantly protected against oxidative stress by illuminating light prior to $\mathrm{H}_{2} \mathrm{O}_{2}$ treatment (Fig. 3B). LDH release and cell detachment were both suppressed by light illumination. The light illumination just before $\mathrm{H}_{2} \mathrm{O}_{2}$ challenge was most effective in protecting cells, indicating that the effect of light activation of cellular Akt was transient and did not last long. In this way, photo-activated Akt was demonstrated to protect cells against biological stresses such as nutritional deprivation and oxidative stress.

In conclusion, the PA-Akt probe phosphorylated/ activated cellular Akt in AML12 cells in a time- and intensity-dependent fashion with regard to light illumination. Though the effect of the PA-Akt probe seemed transient, it conferred resistance against various physiopathological stresses. Studies are ongoing to evaluate the efficacy of this probe against other types of stresses such as ligand/signal-mediated cell death. Therefore, the optimal conditions must be examined to induce and sustain sufficient Akt activity according to the purposes and experiments. The PA-Akt probe can be a unique and useful tool to study Akt-associated cell functions and to develop anticancer drugs.

ACKNOWLEDGMENTS: This work was supported by the JSPS Grants-in-Aid for Scientific Researches (KAKENHI) [Grant Nos. 26220805 (to M.O.), 26670573 and $15 H 05659$ (to S.H.), and $17 K 19877$ (to M.A.)]. This work was also supported in part by the Grants-in-Aid for Regional R\&D ProposalBased Program from the Northern Advancement Center for Science and Technology of Hokkaido Japan (to M.O.), the Takeda Science Foundation (to S.H.), a research grant from The Akiyama Life Science Foundation (to S.H.), and a donation from Mr. and Mrs. Fujikawa (to M.O.). The authors declare no conflicts of interest.

\section{REFERENCES}

1. Nakano N, Matsuda S, Ichimura M, Minami A, Ogino M, Murai T, Kitagishi Y. PI3K/AKT signaling mediated by $G$ protein-coupled receptors is involved in neurodegenerative Parkinson's disease. Int J Mol Med. 2017;39:253-60.
2. Zhao EY, Efendizade A, Cai L, Ding Y. The role of Akt (protein kinase B) and protein kinase $\mathrm{C}$ in ischemia-reperfusion injury. Neurol Res. 2016;38:301-8.

3. Haga S, Ogawa W, Inoue H, Terui K, Ogino T, Igarashi R, Takeda K, Akira S, Enosawa S, Furukawa H, Todo S, Ozaki M. Compensatory recovery of liver mass by Akt-mediated cellular hypertrophy in liver-specific STAT3-deficient mice. J Hepatol. 2005;43:799-807.

4. Haga S, Ozaki M, Inoue H, Okamoto Y, Ogawa W, Takeda $\mathrm{K}$, Akira S, Todo S. The survival pathways phosphatidylinositol-3 kinase (PI3-K)/phosphoinositide-dependent protein kinase 1 (PDK1)/Akt modulate liver regeneration through hepatocyte size rather than proliferation. Hepatology 2009;49:204-14.

5. Inoue $H$, Ogawa $W$, Ozaki $M$, Haga $S$, Matsumoto $M$, Furukawa K, Hashimoto N, Kido Y, Mori T, Sakaue H, Teshigawara K, Jin S, Iguchi H, Hiramatsu R, LeRoith D, Takeda K, Akira S, Kasuga M. Role of Stat-3 in regulation of hepatic gluconeogenic genes and carbohydrate metabolism in vivo. Nat Med. 2004;10:168-74.

6. Wang $\mathrm{C}$, Yao B, Xu M, Zheng X. RIP1 upregulation promoted tumor progression by activating AKT/Bcl-2/BAX signaling and predicted poor postsurgical prognosis in HCC. Tumour Biol. 2016;37:15305-13.

7. Liu D, Zhang Y, Wei Y, Liu G, Liu Y, Gao Q, Zou L, Zeng W, Zhang N. Activation of AKT pathway by Nrf2/PDGFA feedback loop contributes to HCC progression. Oncotarget 2016;7:65389-402.

8. Hou YQ, Yao Y, Bao YL, Song ZB, Yang C, Gao XL, Zhang WJ, Sun LG, Yu CL, Huang YX, Wang GN, Li YX. Juglanthraquinone $\mathrm{C}$ induces intracellular ROS increase and apoptosis by activating the Akt/Foo signal pathway in HCC cells. Oxid Med Cell Longev. 2016;2016:4941623.

9. Sui Y, Zheng X, Zhao D. Rab31 promoted hepatocellular carcinoma (HCC) progression via inhibition of cell apoptosis induced by PI3K/AKT/Bcl-2/BAX pathway. Tumour Biol. 2015;36:8661-70.

10. Zhou SL, Zhou ZJ, Hu ZQ, Li X, Huang XW, Wang Z, Fan J, Dai Z, Zhou J. CXCR2/CXCL5 axis contributes to epithelial-mesenchymal transition of HCC cells through activating PI3K/Akt/GSK-3 $\beta /$ Snail signaling. Cancer Lett. 2015;358:124-35.

11. Cheng GZ, Park S, Shu S, He L, Kong W, Zhang W, Yuan Z, Wang LH, Cheng JQ. Advances of AKT pathway in human oncogenesis and as a target for anti-cancer drug discovery. Curr Cancer Drug Targets 2008;8:2-6.

12. Mitsiades CS, Mitsiades N, Koutsilieris M. The Akt pathway: Molecular targets for anti-cancer drug development. Curr Cancer Drug Targets 2004;4:235-56.

13. Katsura Y, Kubota H, Kunida K, Kanno A, Kuroda S, Ozawa T. An optogenetic system for interrogating the temporal dynamics of Akt. Sci Rep. 2015;5:14589. 